\title{
Mental state attribution and body configuration in women
}

\author{
Jennifer A. Bremser ${ }^{1,2}$ * and Gordon G. Gallup Jr. ${ }^{1}$ \\ Department of Psychology, University at Albany, Albany, NY, USA \\ 2 Department of Psychology, Student Development Center, Alfred State College, Alfred, NY, USA
}

Edited by:

Melanie L. Shoup-Knox, University at Albany, USA

\section{Reviewed by:}

Melanie L. Shoup-Knox, University at Albany, USA

Will Lassek, University of Pittsburgh Graduate School of Public Health, USA

\section{*Correspondence:}

Jennifer A. Bremser, Department of Psychology, Student Development Center, Alfred State College, Alfred, NY 14802, USA.

e-mail: bremseja@alfredstate.edu
Body configuration is a sexually dimorphic trait. In humans, men tend to have high shoulderto-hip ratios. Women in contrast, often have low waist-to-hip ratios (WHR); i.e., narrow waists and broad hips that approximate an hour-glass configuration. Women with low WHR's are rated as more attractive, healthier, and more fertile. They also tend to have more attractive voices, lose their virginity sooner, and have more sex partners. WHR has also been linked with general cognitive performance. In the present study we expand upon previous research examining the role of WHR in cognition. We hypothesized that more feminine body types, as indexed by a low WHR, would be associated with cognitive measures of the female "brain type," such as mental state attribution and empathy because both may depend upon the activational effects of estrogens at puberty. We found that women with low WHRs excel at identifying emotional states of other people and show a cognitive style that favors empathizing over systemizing. We suggest this relationship may be a byproduct of greater gluteofemoral fat stores which are high in the essential fatty acids needed to support brain development and cellular functioning. It is interesting to note that our findings suggest lowerWHR females, who are more likely to be targeted for dishonest courtship, may be better at identifying disingenuous claims of commitment.

Keywords: mental state attribution, empathy, waist-to-hip ratio, gluteofemoral fat, honest and dishonest courtship

\section{INTRODUCTION}

Some of the more notable sex differences among humans in body configuration are found in the abdominal and gluteofemoral regions. The sexually dimorphic distribution of body fat and pelvic skeletal features are influenced by sex hormones, which explains why the differences in body configuration do not appear until puberty (Singh, 1993). Estrogen inhibits fat deposits in the abdominal region and stimulates deposits in the gluteofemoral region of the body. Conversely, testosterone stimulates deposits in the abdominal region and inhibits fat deposits in the gluteofemoral region.

Men tend to have high shoulder-to-hip ratios; i.e., broad shoulders and narrow hips that create a wedge shaped torso (e.g., Hughes et al., 2004). Women in contrast, often have low waist-to-hip ratios (WHR's); i.e., narrow waists and broad hips that approximate an hour-glass configuration (Singh, 1993). Until recently, the adaptive significance of a low WHR had been viewed in light of its relationship to health and fertility, and linked to perceptions of attractiveness in women (Van Noord-Zaadstra et al., 1991; Zaadstra et al., 1993; Wass et al., 1997; Moran et al., 1999; Westman and Marlowe, 1999; Furnham et al., 2002; Swami et al., 2006; Dixson et al., 2007; Lassek and Gaulin, 2007; Platek and Singh, 2010). Recent evidence shows that low WHR may offer cognitive advantages as well. Gluteofemoral fat in close proximity to the hips is high in long chain polyunsaturated fatty acids (LCPUFAs), including docosahexaenoic acid (DHA; Pittet et al., 1979; Phinney et al., 1994). These chains make up a substantial portion of all cell membranes, and comprise structural components of the brain and retina. They are especially important during fetal development, because the fetus cannot synthesize LCPUFAs and the only source of these nutrients is from maternal supplies (Van Houwelingen et al., 1999). When the dietary intake of essential fatty acids is inadequate, sources stored in adipose tissue is used as an additional supply (Hornstra et al., 1995). Because of the relationship between gluteofemoral fat and LCPUFAs, women with lower WHR's (narrow waists and more accentuated hips) would be expected to have larger stores of these essential fatty acid chains.

Lassek and Gaulin (2008) showed that WHR was inversely related to measures of performance on general cognitive tests in women and their offspring. WHR accounted for $7 \%$ of the variance in the mother's scores and $2.7 \%$ of the variance in their offspring's scores. In cases of teenage pregnancies, the mother's brain is still developing and as a result, developing offspring compete with their mother for brain building resources. They also demonstrated that greater gluteofemoral fat stores can act as a protective buffer. The offspring of teen mothers with lower WHR's were protected against the cognitive deficits that are often associated with teen pregnancy and childbirth (Lassek and Gaulin, 2008). The cognitive advantages of low WHR are further evidenced by studies which show that higher WHR's in both males and females are associated with poor outcomes on cognitive tests (Jagust et al., 2005; Waldstein and Katzel, 2006) and may be associated with age related cognitive declines, particularly in females (Han et al., 2009). Lassek and Gaulin (2008) propose that the reason low WHR is a proximate marker of attractiveness is because greater stores of gluteofemoral fat provide potential cognitive benefits to their offspring.

It is less clear how WHR relates to other domain specific aspects of cognition. We reasoned that WHR may be related to aspects of cognition that are known to be sexually dimorphic, including elements of social intelligence such as theory of mind (ToM) and 
empathy. Mental state attribution, or ToM, is the ability to infer the mental states of other people in terms of what they may know, want, or intend to do (Baron-Cohen, 2001). Empathizing makes use of these attributions to identify the emotional states of others and to respond to these states with the appropriate emotion. On tasks that measure empathizing and mental state attribution, females have an advantage, particularly when it comes to empathizing (Baron-Cohen, 2002). Puberty, when the sexual dimorphisms in WHR become noticeable, may represent an important period for the development of mental state attribution. During and after the onset of puberty, there might have been selective pressures on females to accurately infer and gage the intentions and emotional states of potential mates.

The most widely used social cognition test to measure individual differences in mental state attribution is the "reading of the mind in the eyes test" (RME), Baron-Cohen et al. (2001). Scores on this test are based on a person's accuracy in attributing a mental state to other's using information based solely on their eyes. Females score slightly higher on this task than males, and noticeably higher than both males and females on the autism spectrum, who are often characterized by ToM or mental state attribution impairments (Baron-Cohen et al., 2001). Intranasal administration of oxytocin, a hormone known for its role in human social cognition including aspects of social memory, perception, emotion recognition, affect sharing, social awareness, and face processing (Bartz et al., 2011), improves scores on the RME in adult males and adolescent males diagnosed with an autism spectrum condition. These studies suggest that oxytocin may enhance performance on social cognitive tasks (Domes et al., 2007; Guastella, 2010). In contrast, testosterone levels negatively affect mental state attribution. van Honk et al. (2011) showed that women given a single oral dose of testosterone had lower scores on the RME. The negative effects of testosterone had the greatest influence on women whose digit ratio indicated greater early prenatal androgen exposure. Thus, they concluded that early exposure to testosterone may exhibit a priming effect whereby greater exposure early in life, increases a person's sensitivity to testosterone later in life. Females score significantly higher on measures of empathizing, and from 1 year of age females respond with more concern to the distress of other people through more sad looks, vocalizations, and comforting behaviors (Hoffman, 1977). In the present study we investigated mental state attribution and a bias toward empathizing as a function of variation in WHRs in a sample of female college students to test the hypothesis that low WHR might be associated with enhanced aspects of social cognition.

\section{MATERIALS AND METHODS SUBJECTS}

A sample of 44 female undergraduates were recruited from psychology courses at the State University of New York at Albany. Participant age ranged between 18 and 22 years (Mean age $=19.1$ $\mathrm{SD}=1.08$ ) and all were fluent in English. The methods used in this study were approved by the campus Institutional Review Board and conformed to guidelines for the ethical treatment of human subjects. The participants were not aware of the purpose of the study. They gave informed consent and received course credit for their participation.

\section{ANTHROPOMETRICS}

Waist-to-hip ratios were obtained using the method employed by Hughes et al. (2004). Waist and hip circumference was measured to the nearest $0.5 \mathrm{~cm}$ using anthropometric fiberglass body measuring tape by one of two female investigators who did not know the participants' responses to other study questions. Waist circumference was defined as the smallest girth between the rib cage and the iliac crest. Hip circumference was defined as the largest girth between the waist and thigh. The inter-rater reliability among both females who measured WHRs was high, $r=0.998$.

\section{MEASURES OF MENTAL STATE ATTRIBUTION, EMPATHIZING, AND SYSTEMIZING}

The RME was used to assess mental state attribution. The RME consists of 36 partial face photographs, showing only the eye region of different actors with different facial expressions. Participants choose which mental state (out of four possible options) that best describes what the person in the photograph is feeling or thinking. The photographs were presented on a computer screen, and participants were provided with a vocabulary key in order to look up the description of the mental states on the test, if needed. Normative data on the RME suggests a slight, but non-significant female advantage on the task (Baron-Cohen et al., 2001).

\section{EMPATHIZING QUOTIENT}

The empathizing quotient (EQ) is a forced choice, self-report questionnaire used to assess different dimensions of empathetic responsiveness (Wheelwright et al., 2006). Structurally, it is comprised of 60 items with 40 items assessing empathy and 20 filler items. Responses are given on a four-point scale depending on how strongly participants agree or disagree with statements related to empathy, and scoring direction is counterbalanced. Females typically score higher than males on this instrument and it can be used to identify individuals with Asperger's syndrome. Scores on the EQ range from 0 to 80 . The instrument has high internal consistency with a Cronbach's alpha of 0.852 , and is considered a valid measure of empathy (Muncer and Ling, 2006). Empathizing appears to be mostly, but not completely, independent of systemizing (Wheelwright et al., 2006).

\section{SYSTEMIZING OUOTIENT}

Systemizing is the drive to identify the if-then correlational rules governing natural, physical, and social systems. Males tend to outperform females on measures of systemizing. The systemizing quotient-revised (SQR) is a 75-item forced choice, self-report questionnaire that measures systemizing ability (Wheelwright et al., 2006). Participants select a response ranging from "strongly agree" to "strongly disagree" with statements concerned with the mechanical, abstract, domestic, and social aspects of systemizing. On average, males score higher than females on the SQR (Wheelwright et al., 2006).

\section{RESULTS}

Data from each participant were entered into a spreadsheet and analyzed using SPSS/PAWS version 18.0. An exploratory analysis revealed that the distribution for scores were normal for every variable. Descriptive statistics for each measure are presented 
in Table 1. Scores on the RME reflect the number of accurate responses (out of 36 possible). Since the EQ and SQR have different numbers of questions, EQ and SQR scores are presented as percentages. A composite variable identified as "Empathizing Bias" was created for each participant by computing the $z$ scores for the EQ and SQR, and then subtracting the participants' SQR $z$-score from the EQ $z$-score $(z$-EQ $-z$-SQR $=$ empathizing bias). A positive empathizing bias reflects the tendency to utilize a cognitive style that favors empathizing over systemizing and this has been supported by other studies (Brosnan et al., 2010). An outlier who scored more than 4 SD away from the mean $(X=-0.0413, \mathrm{SD}=1.1)$ on this variable was removed from subsequent analyses.

Descriptive statistics are provided in Table 1. A Pearson product moment correlation between WHR and performance on the RME task was significant $(r=-0.449 p<0.001)$. WHR also explained a significant proportion of variance in scores on the RME, $R^{2}=0.201, F(1,42)=10.59, p<0.01$. In addition, we found a significant correlation between WHR and empathizing bias $(r=-0.296, p<0.05)$. Correlations between WHR and systemizing were not significant.

\section{DISCUSSION}

Our data show that more feminine body types were associated with dimensions of the female brain including a superior ability to read emotions in faces. As shown in Figure 1, variation in WHRs among the women in this sample accounted for over $20 \%$ of the observed differences in performance on the RME. In addition, we found that lower waist-to-hips ratios were also associated with empathizing bias. This provides convergent evidence that females with more accentuated hour-glass figures are advantaged on tasks related to mental state attribution, and the presence of a cognitive style that favors empathizing over systemizing indicates that the relationship between WHR and performance on the Eyes in the Mind task was not spurious.

Gluteofemoral fat may contribute to this advantage. The most notable sex differences in the accumulation of body fat are observed in the abdominal and gluteofemoral regions. The sexually dimorphic distribution of body fat is influenced by sex hormones, which explains why the differences in body configuration do not appear until puberty (Singh, 1993). Estrogen inhibits fat deposits in the abdominal region and stimulates deposits in the gluteofemoral region of the body. Therefore, estrogen is a critical factor differentiating women with high WHR from women with low WHR. Conversely, testosterone promotes deposits in the abdominal region and inhibits fat deposits in the gluteofemoral

Table 1 | Descriptive statistics for study variables.

\begin{tabular}{llll}
\hline & Mean & SD & Range \\
\hline Waist-to-hip ratio & 0.72 & 0.06 & $0.62-0.93$ \\
Reading the mind in the eyes & 26.4 & 4.1 & $19-34$ \\
Empathy quotient & $47.2(59 \%)$ & 10.1 & $28-69$ \\
Systemizing quotient & $56.9(38 \%)$ & 15.6 & $31-90$ \\
Empathizing bias & 0.56 & 0.87 & -1.45 to 2.11
\end{tabular}

region. In general, women have lower WHR's than men; however, after menopause, a woman's WHR increases. This is because, as women age, the ratio of androgens to estrogens increases (Kirschner and Samojlik, 1991). Because the distribution of body fat is influenced by sex hormones, this has important implications for sex differences in cognition. Schattman and Sherwin (2007) studied females with polycystic ovary syndrome, a condition leading to higher levels of testosterone levels, and found that women with polycystic ovary syndrome performed worse on verbal fluency and verbal memory tasks, suggesting that testosterone may interfere with cognitive tasks where females generally show an advantage.

Among girls of the same body weight, those with lower WHR's experience earlier pubertal endocrine activity. When body weight, height, pubic hair growth, age, and pelvic breadth are controlled for, girls with the lowest WHR's have the highest levels of estrogen and gonadotrophins, luteinizing hormone, and follicle stimulating hormone (deRidder et al., 1990). Lassek and Gaulin (2008) provide evidence that females with low WHR and their offspring show improved performance on general cognitive tests. Other studies have also shown that high WHR's among the elderly are correlated with poor cognitive performance and brain changes (Jagust et al., 2005; Waldstein and Katzel, 2006).

Waist-to-hip ratio is a measure of both pelvic skeletal morphology and soft tissue features. The soft tissue differences are largely a consequence of underlying differences in fat stores. Low WHR's imply that there is more fat in the gluteal region (characteristic of females), while high WHR's are associated with more fat in visceral and abdominal regions (characteristic of males). Female's tendency to carry greater fat stores than males may function to offset the energetic costs associated with pregnancy and lactation (Dufour and Slather, 2002).

It is interesting to note that gluteal adipose tissue is a major source of long-chain polyunsaturated fatty acids (LCPUFAs), such as omega-3 DHA. LCPUFAs make up roughly $20 \%$ of the dry matter of neurons (Hachey et al., 1987; Demmelmair et al., 1998; Del Prado et al., 2000; Fidler et al., 2000), and increases in maternal DHA intake appear to have beneficial effects on offspring IQ scores (Cohen et al., 2005; Hibbeln et al., 2007).

Low WHR females have larger stores of LCPUFAs than high WHR females because gluteofemoral fat stores are higher in LCPUFAs than abdominal and visceral fat (Pittet et al., 1979; Schafer and Overvad, 1990; Phinney et al., 1994). Low WHR gluteofemoral fat is also associated with high levels of DHA (Desci et al., 1996; Garaulet et al., 2001), while high WHR's decrease DHA concentrations (Hollmann et al., 1997; Decsi et al., 2000).

Previous research has documented the adaptive significance of WHR in terms of its relationship to fertility, health, and general cognition. Our data suggest that WHR may have important and more domain specific implications for social intelligence. In contrast to what Lassek and Gaulin (2008) found for general cognitive tests where WHR accounted for $7 \%$ of the variance in performance, our data show that WHR accounts for almost three times more variance in performance on a more specific test of mental state attribution. Females with low WHR's who excel at making mental state attributions may benefit in several ways. First, mental state attribution may be an important component to mate 


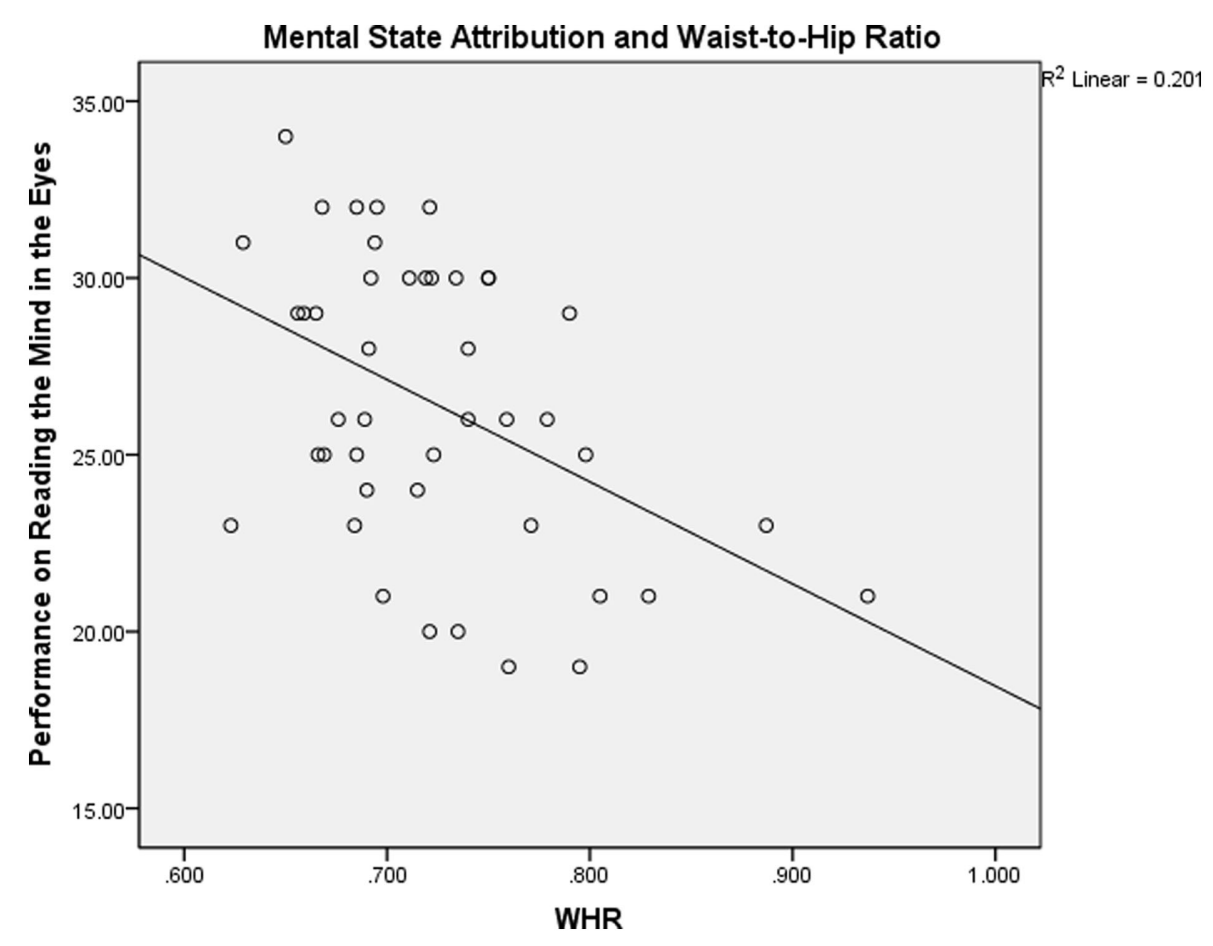

FIGURE 1 | Mental state attribution as a function of waist-to-hip ratios.

selection. Most studies of WHR and attractiveness find that men prefer women with lower WHR's (Singh et al., 2010). Even congenitally blind men display a preference for low WHR (Karremans et al., 2010). In addition, women with low WHR's have more attractive voices, lose their virginity sooner, have more sex partners, and are more prone to engage in extra-pair copulations (Hughes et al., 2004).

Females with low WHR's who excel at mental state attribution may be able to better distinguish between males who feign good intentions for purposes of gaining sexual favors (e.g., "I love you, so let's go to bed") and those with honest intentions to enter into a long-term committed relationship. Because low WHR females might be pursued more actively by males using both kinds of strategies, there may have been selective pressure to accurately gage the intentions of prospective mates as a means of reducing the likelihood of falling victim to dishonest courtship strategies. In line with this idea, our data show that WHR is inversely related to mental state attribution in females, suggesting that lower WHR females who are more likely to be targeted for dishonest courtship are better at distinguishing between genuine and disingenuous claims of commitment.

Finally, it is important to note that mental state attribution and empathy also have implications for effective child rearing. Our results suggest that women with low WHR's may also be better at responding to signs of distress on the part of their children and adopting more timely and effective intervention strategies.

Waist-to-hip ratio may also have important implications for age related declines in cognition. Females WHR declines with age (Tichet et al., 1993). Each episode of pregnancy and lactation draws from the mother's gluteofemoral fat stores, and in theory, this leaves less cognitive maternal resources to protect against age related declines in mental function. Based on what is known about WHR and cognition, we would predict that as the number of offspring increase there ought to be a concomitant decline in cognition. Indeed, number of offspring is related to the likelihood of developing dementia among women later in life (Ptok et al., 2002; Beeri et al., 2009). Of particular interest is the fact that Alzheimer's disease is associated not only with declines in general cognition but also with specific deficits in ToM (Zaitchik et al., 2006).

It is important to acknowledge that our study has several limitations. First, we relied on scores from the Reading the Mind in the Eyes test as a measure of mental state attribution. Although, this measure is a widely used and reliable instrument it may not completely capture the entire range of social cognitive processes involved in making mental state attributions. The images used in this test are static, whereas social interactions in the real world are usually dynamic. The RME relies on accuracy data (the number of correct responses) but it is not sensitive to the different types of errors that can be made. For instance, there can be errors of absence (i.e., failing to detect an emotional state when it is present) or errors of excess (falsely inferring an emotional state when it is absent). Previous research has shown that at least some types of mental state misattributions are themselves, a sexual dimorphic feature of social cognition; for example, males are more likely to infer sexual interest from females that are not genetically related to them (Haselton and Buss, 2000). Future research could examine how WHR relates to social cognition using more sensitive measures of mental state attribution. 


\section{REFERENCES}

Baron-Cohen, S. (2001). Theory of mind and autism: a review. Int. Rev. Res. Ment. Retard. 23, 169.

Baron-Cohen, S. (2002). The extreme male brain theory of autism. Trends Cogn. Neurosci. (Regul. Ed.) 6, 248-254.

Baron-Cohen, S., Wheelwright, S., Hill, J., Raste, Y., and Plumb, I. (2001). The "reading the mind in the eyes" test revised version: a study with normal adults, and adults with asperger syndrome or high-functioning autism. J. Child. Psychol. Psychiatry 42, 2, 241-251.

Bartz, J. A., Zaki, J., Bolger, N., and Ochsner, K. N. (2011). Social effects of oxytocin in humans: context and person matter. Trends Cogn. Sci. (Regul. Ed.) 15, 301-309.

Beeri, M. S., Rapp, M., Schmeidler, J., Reichenberg, A., Purohit, D. P., Perl, D. P., Grossman, H. T., Prohovnik, I., Haroutunian, V., and Silverman, J. M. (2009). Number of children is associated with neuropathology of Alzheimer's disease in women. Neurobiol. Aging 30, 1184-1191.

Brosnan, M., Aschwin, C., Walker, I., and Donahue, J. (2010). Can an "extreme female brain" be characterized in terms of psychosis? Pers. Individ. Dif. 49, 738-743.

Cohen, J. T., Bellinger, D. C., Connor, W. E., and Shaywitz, B. A. (2005). A quantitative analysis of prenatal intake of n-3 polyunsaturated fatty acids and cognitive development. Am. J. Prev. Med. 29, 366-374.

Del Prado, M., Villapndo, S., Lance, A., Alfonso, E., Demmelmair, H., and Koletzko, B. (2000). Contribution of dietary and newly formed arachidonic acid to milk secretion in women in low fat diets. Adv. Exp. Med. Biol. 478, 407-408.

Demmelmair, H., Baumheur, M., Koletzko, B., Dokupil, K., and Kratl, G. (1998). Metabolism on U13Clabeled linoleic acid in lactating women. J. Lipid Res. 39, 1389-1396.

Decsi, T., Csabi, G., Torok, K., Erhadt, E., Minda, H., Burus, I., Molnar, S., and Molnar, D. (2000). Polyunsaturated fatty acids in plasma of obese children with and without metabolic cardiovascular syndrome. Lipids 35, 1179-1184.

Desci, T., Molnar, D., and Koletzko, B. (1996). Long-chain polyunsaturated fatty acids in plasma lipids of obese children. Lipids 31, 305-311.

deRidder, C. M., Bruning, P. F., Zonderland, M. L., Thijssen, J. H., Bonfer, J. M., Blankenstein, M. A., Huisveld,
I. A., and Erich, W. B. (1990). Body fat mass, body fat distribution, and plasma hormones in early puberty females. J. Clin. Endocrinol. Metab. 70, 888-893.

Dixson, B. J., Dixson, A. F., Li, B., and Anderson, M. J. (2007). Studies of human physique and sexual attractiveness: sexual preferences of men and women in China. Am. J. Hum. Biol. 19, 88-95.

Domes, G., Heinrichs, M., Michel, A., Berger, C., and Herpertz, S. C. (2007). Oxytocin improves "mindreading" in humans. Biol. Psychiatry 61, 731-733.

Dufour, D. L., and Slather, M. L. (2002). Comparative and evolutionary dimensions of the energetics of human pregnancy and lactation. Am. J. Hum. Biol. 14, 584-602.

Fidler, N., Sauerwald, T., Pohl, A., Demmelmair, H., and Koletzko, B. (2000). Docosahexanoic acid transfer into human milk after supplementation: a randomized clinical trial. J. Lipid. Res. 41, 1376-1383.

Furnham, A., Moutafi, J., and Baguma, B. (2002). A cross-cultural study on the role of weight and waist-to-hip ratio on female attractiveness. Pers. Individ. Dif. 32, 729-745.

Garaulet, M., Perez-Llamas, F., PerezAyala, M., Martinez, P., de Medina, F. S., Tebar, F. J., and Zamora, S. (2001). Site-specific differences in the fatty acid composition of abdominal adipose tissue in an obese population from the Mediterranean area. Am. J. Clin. Nutr. 74, 585-591.

Guastella, A. J. (2010). Intranasal oxytocin improves emotion recognition for youth with autism spectrum disorders. Biol. Psychiatry 67, 692-694.

Hachey, D. L., Thomas, R. M., Emken, E. A., Garza, C., Brown-Booth, L., Adolf, R. O., and Klein, P. D. (1987). Human lactation: maternal transfer of dietary triglycerides labeled with stable isotopes. J. Lipid Res. 28, 1185-1192.

Han, C., Jo, S. A., Seo, J. A., Kim, B. G., Kim, N. H., Jo, I., Park, M. H., and Park, K. W. (2009). Adiposity parameters and cognitive function in the elderly: application of the jolly fat hypothesis to cognition. Arch. Gerontol. Geriatr. 49, 2, 133-138.

Haselton, M. G., and Buss, D. M. (2000). Error management theory: a new perspective on cross-sex mind reading. J. Pers. Soc. Psychol. 78, 81-91.

Hibbeln, J. R., Davis, J. M., Steer, C., Emmett, P., Rogers, I., Williams, C., and Golding, J. (2007). Maternal seafood consumption in pregnancy and neurodevelopmental outcomes in childhood (ALSPAC study): an observational cohort study. Lancet 369, 578-585.

Hoffman, M. L. (1977). Sex differences in empathy and related behaviors. Psychol. Bull. 84, 712-722.

Hollmann, M., Runnebaum, B., and Gerhard, I. (1997). Impact of waistto-hip and body mass index on hormonal and metabolic parameters in young, obese women. Int. J. Obes. 21 , 476-483.

Hornstra, G., Al, M., Houwelingen, A., and Foreman-Van Drongelen, M. (1995). Essential fatty acids in pregnancy and early human development. Eur. J. Obstet. Gynecol. Reprod. Biol. 61, 57-62.

Hughes, S. M., Dispenza, F., and Gallup, G. G. Jr. (2004). Ratings of voice attractiveness predict sexual behavior and body configuration. Evol. Hum. Behav. 25, 295-304.

Jagust, W., Harvey, D., Mungas, D., and Haan, M. (2005). Central obesity and the aging brain. Arch. Neurol. 62, 1545-1548.

Karremans, J. C., Frankenhuis, W. E., and Arons, S. (2010). Blind men prefer a low waist-to-hip ratio. Evol. Hum. Behav. 31, 182-186.

Kirschner, M. A., and Samojlik, E. (1991). Sex hormone metabolism in upper and lower body obesity. Int. J. Obes. 15, 101-108.

Lassek, W. D., and Gaulin, S. J. C. (2007). Menarche is related to fat distribution. Am. J. Phys. Anthropol. 133, 1147-1151.

Lassek, W. D., and Gaulin, S. J. C. (2008). Waist-to-hip and cognitive ability: is gluteofemoral fat a privileged store of neurodevelopmental resources? Evol. Hum. Behav. 29, 26-34.

Moran, C., Hernandez, E., Ruiz, J. E., Fonseca, M. E., Bermudez, J. A., and Zarate, A. (1999). Upper body obesity and hyperinsulinemia are associated with anovulation. Gynecol. Obstet. Invest. 47, 1-5.

Muncer, S., and Ling, J. (2006). Psychometric analysis of the empathy quotient. Pers. Individ. Dif. 20, 1111-1119.

Phinney, S. D., Stern, J. S., Burke, K. E., Tang, A. B., Miller, G., and Holman, R. (1994). Human subcutaneous adipose tissue shows sitespecific differences in fatty acid composition. Am. J. Clin. Nutr. 60, 725-729.

Pittet, P. G., Halliday, D., and Bateman, P. E. (1979). Site differences in the fatty acid composition of subcutaneous adipose tissue of obese women. Br. J. Nutr. 42, 57-61.
Platek, S. M., and Singh, D. (2010). Optimal waist to hip ratios activate neural reward centers in men. PLoS ONE 5, e9042. doi:10.1371/journal.pone.0009042

Ptok, U., Barkow, K., and Heun, R. (2002). Fertility and number of children in patients with Alzheimer's disease. Arch. Womens Ment. Health 5, 83-86.

Schafer, L., and Overvad, K. (1990). Subcutaneous adipose-tissue fatty acids and vitamin $\mathrm{E}$ in humans: relation to diet and sampling site. Am. J. Clin Nutr. 52, 486-490.

Schattman, L., and Sherwin, B. B. (2007). Effects of pharmacologic manipulation of testosterone on cognitive functioning in women with polycystic ovary syndrome: a randomized placebo-controlled treatment study. Horm. Behav. 51, 579-686.

Singh, D. (1993). Adaptive significance of female physical attractiveness: role of waist - hip ratio. J. Pers. Soc. Psychol. 65, 293-307.

Singh, D., Dixson, B. J., Jessop, T. S., Morgan, B., and Dixson, A. F. (2010). Cross-cultural consensus for waist-to-hip ratio and women's attractiveness. Evol. Hum. Behav. 31, 176-181.

Swami, V., Caprario, C., Tovee, M. J., and Furnham,A. (2006). Female physical attractiveness in Britain and Japan: a cross-cultural study. Eur. J. Pers. 20, 69-81.

Tichet, J., Vol, S., Balkau, B., Le Clesiau, H., and D'Hour, A. (1993). Android fat distribution by age and sex: the waist hip ratio. Diabete. Metab. 19 , 273-276.

van Honk, J., Schutter, D. J., Bos, P. A., Kruitjt, A., Lentjes, E. G., and Baron-Cohen, S. (2011). Testosterone administration impairs cognitive empathy in women depending on second-to-fourth digit ratio. Proc. Natl. Acad. Sci. U.S.A. 108, 3448-3452.

Van Houwelingen, A. C., Ham, E. C., and Hornstra, G. (1999). The female docosahexaenoic acid status related to the number of completed pregnancies. Lipids 34, S229.

Van Noord-Zaadstra, B. M., Seidell, J. C., Vrieswijk, B., and Van Noord, P. A. H. (1991). The relationship between fat distribution and fertility: a prospective study of healthy Dutch women. Int. J. Obes. 15(Suppl. 3), 36 .

Waldstein, S. R., and Katzel, L. I. (2006). Interactive relations of central versus total obesity and blood pressure to cognitive function. Int. J. Obes. 30 , 201-207. 
Wass, P., Waldenstrom, U., Rossner, S., and Hellberg, D. (1997). An android body fat distribution in females impairs pregnancy rate of in-vitro fertilization - embryo transfer. Hum. Reprod. 12, 2057-2060.

Westman, A., and Marlowe, F. (1999). How universal are preferences for female waist-to-hip ratios? Evidence from the Hadza of Tanzania. Evol. Hum. Behav. 20, 219-228.

Wheelwright, S., Baron-Cohen, S., Goldenfeld, N., Fine, D., Smith, R., Weil, L., and Wakabayashi, A.
(2006). Predicting autism spectrum quotient (AQ) from the systemizing quotient-revised (SQ-R) and empathy quotient (EQ). Brain Res. 1079, 47-56.

Zaadstra, B. M., Seidell, J. C., Van Noord, P. A., te Velde, E. R., Habbema, J. D., Vrieswijk, B., and Karbaat, J. (1993). Fat and female fecundity: prospective study of effect of body fat distribution on conception rates. BMJ 306, 484-487.

Zaitchik, D., Koff, E., Brownell, H., Winner, E., and Albert, M. (2006).
Inference of beliefs and emotions in patients with Alzheimer's disease. Neuropsychology 20, 11-20.

Conflict of Interest Statement: The authors declare that the research was conducted in the absence of any commercial or financial relationships that could be construed as a potential conflict of interest.

Received: 15 August 2011; accepted: 03 January 2012; published online: 30 January 2012.
Citation: Bremser JA and Gallup GG Jr. (2012) Mental state attribution and body configuration in women. Front. Evol. Neurosci. 4:1. doi 10.3389/fnevo.2012.00001

Copyright (c) 2012 Bremser and Gallup Jr.. This is an open-access article distributed under the terms of the Creative Commons Attribution Non Commercial License, which permits non-commercial use, distribution, and reproduction in other forums, provided the original authors and source are credited. 